\title{
The hemoglobin adduct $N$-(2,3-dihydroxypropyl)-valine as biomarker of dietary exposure to glycidyl esters: a controlled exposure study in humans
}

\author{
Klaus Abraham ${ }^{1} \cdot$ Jan Hielscher ${ }^{1} \cdot$ Tobias Kaufholz $^{2} \cdot$ Hans Mielke $^{2} \cdot$ Alfonso Lampen $^{1} \cdot$ Bernhard Monien $^{1}$
}

Received: 17 October 2018 / Accepted: 4 December 2018 / Published online: 10 December 2018

(c) The Author(s) 2018

\begin{abstract}
Fatty acid esters of glycidol (glycidyl esters) are heat-induced food contaminants predominantly formed during industrial deodorization of vegetable oils and fats. After consumption, the esters are digested in the gastrointestinal tract, leading to a systemic exposure to the reactive epoxide glycidol. The compound is carcinogenic, genotoxic and teratogenic in rodents, and rated as probably carcinogenic to humans (IARC group 2A). Assessment of exposure from occurrence and consumption data is difficult, as lots of different foods containing refined oils and fats may contribute to human exposure. Therefore, assessment of the internal exposure using the hemoglobin adduct of glycidol, $N$-(2,3-dihydroxypropyl)-valine (2,3-diHOPr-Val), may be promising, but a proof-of-principle study is needed to interpret adduct levels with respect to the underlying external exposure. A controlled exposure study was conducted with 11 healthy participants consuming a daily portion of about $36 \mathrm{~g}$ commercially available palm fat with a relatively high content of ester-bound glycidol ( $8.7 \mathrm{mg}$ glycidol $/ \mathrm{kg})$ over 4 weeks (total amount $1 \mathrm{~kg}$ fat, individual doses between 2.7 and $5.2 \mu \mathrm{g} / \mathrm{kg}$ body weight per day). Frequent blood sampling was performed to monitor the 2,3-diHOPr-Val adduct levels during formation and the following removal over 15 weeks, using a modified Edman degradation and ultrahigh performance liquid chromatography-tandem mass spectrometry (UHPLC-MS/ MS). Results demonstrated for the first time that the relatively high exposure during the intervention period was reflected in corresponding distinct increases of 2,3-diHOPr-Val levels in all participants, following the expected slope for hemoglobin adduct formation and removal over time. The mean adduct level increased from 4.0 to $12.2 \mathrm{pmol} 2,3$-diHOPr-Val/g hemoglobin. By using a nonlinear mixed model, values for the adduct level/dose ratio $(k$, mean $0.082 \mathrm{pmol} 2,3-\mathrm{diHOPr}-\mathrm{Val} / \mathrm{g}$ hemoglobin per $\mu \mathrm{g}$ glycidol/kg body weight) and the adduct lifetime ( $\tau$, mean 104 days, likely the lifetime of the erythrocytes) were determined. Interindividual variability was generally low. 2,3-DiHOPr-Val was therefore proven to be a biomarker of the external dietary exposure to fatty acid esters of glycidol. From the background adduct levels observed in our study, a mean external glycidol exposure of $0.94 \mu \mathrm{g} / \mathrm{kg}$ body weight was estimated. This value is considerably higher than current estimates for adults using occurrence and consumption data of food. Possible reasons for this discrepancy are discussed (other oral or inhalational glycidol sources, endogenous formation, exposure to other chemicals also forming the adduct 2,3-diHOPr-Val). Further research is necessary to clarify the issue.
\end{abstract}

Keywords Glycidol $\cdot$ Edman degradation $\cdot$ Protein adduct $\cdot$ Biomarker $\cdot$ Exposure study

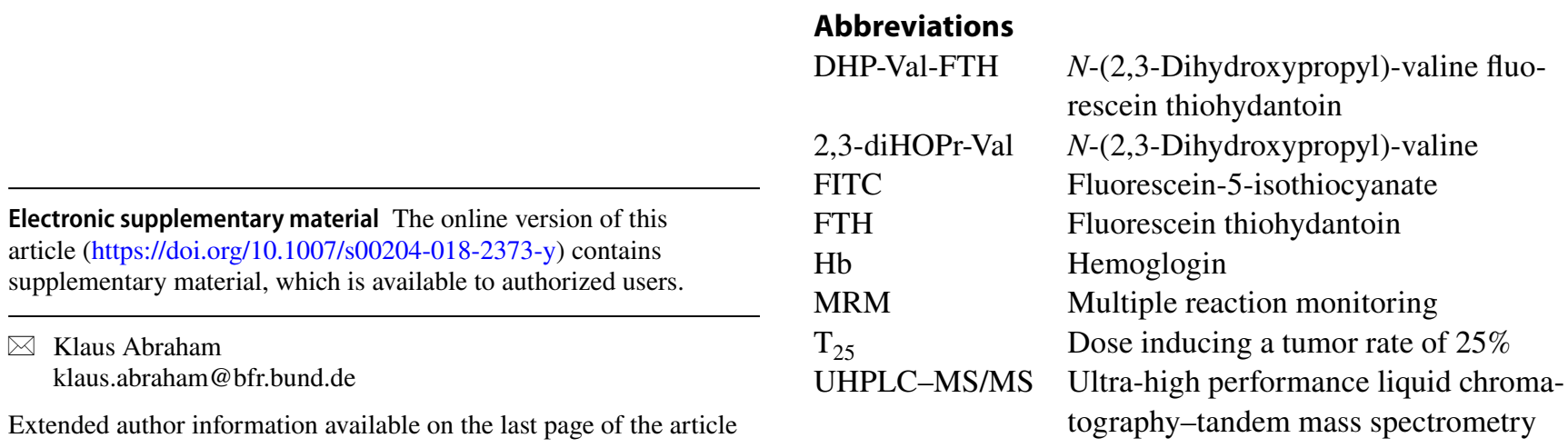




\section{Introduction}

Despite that industrial deodorization of vegetable oils and fats has been used to remove unwanted compounds since more than 100 years, the simultaneous generation of unwanted glycidyl fatty acid esters (and 2-/3-monochloropropanediol esters) - due to the use of high temperatures during the processes-was discovered just about 10 years ago (Svejkovska et al. 2004). Since then, research has been initiated on industrial mitigation strategies on the one hand, and on toxicological properties of these heat-induced food contaminants on the other hand. As demonstrated in rats, glycidyl esters are nearly completely digested in the gastrointestinal tract, leading to a systemic exposure to the reactive epoxide glycidol (Appel et al. 2013; Wakabayashi et al. 2012). The substance is a genotoxic multisite carcinogen (National Toxicology Program 1990) as well as a teratogen (Slott and Hales 1985) in rodents, and rated as probably carcinogenic to humans [IARC group 2A (International Agency for Research on Cancer 2000)]. As for other genotoxic compounds, the margin of exposure (MoE) concept is used in risk assessment. As the reference point, a $\mathrm{T}_{25}$ of $10.2 \mathrm{mg}$ glycidol/ $\mathrm{kg}$ body weight per day was selected for the occurrence of peritoneal mesothelioma in male rats (European Food Safety Authority 2016; Federal Institute of Risk Assessment (BfR) 2009). Accordingly, from a public health point of view, the daily exposure might be considered to be of low concern when it does not exceed $0.408 \mu \mathrm{g} / \mathrm{kg}$ body weight ( $\mathrm{T}_{25}$ based MoE higher that 25,000).

With regard to the occurrence in food, levels of esterbound glycidol were reported to be highest in palm oils or fats (mean $3955 \mu \mathrm{g} / \mathrm{kg}, n=498$ ), but most refined vegetable oil/fats were found to contain substantial quantities. As a major contributor to the exposure to glycidol, mean levels of bound glycidol in margarine were reported to be $582 \mu \mathrm{g} / \mathrm{kg}$ (normal fat, $n=73$ ) and $209 \mu \mathrm{g} / \mathrm{kg}$ (low fat, $n=82$ ). Across all dietary surveys and age groups, exposure values to glycidol were calculated to be $0.1-0.9$ and $0.2-2.1 \mu \mathrm{g} / \mathrm{kg}$ body weight per day for mean and high (P95) exposure in Europe, respectively (European Food Safety Authority 2016). The accuracy of the exposure calculations may be limited due to various uncertainties. First of all, analytical data on glycidyl ester contents are not available for some food classes. In addition, the exposure calculations did not consider the possible formation of glycidyl esters by domestic heat processing of food, e.g., by preparation of meat dishes at $T>200{ }^{\circ} \mathrm{C}$ (Inagaki et al. 2016).

An alternative to the external exposure estimation (which has to consider a huge number of foods possibly containing bound glycidol) may be the quantification of a biomarker allowing monitoring of the individual internal exposure, regardless of the source and route of exposure. The medium-term exposure to glycidol may be determined by the protein adduct $N$-(2,3-dihydroxypropyl)-valine (2,3-diHOPr-Val) formed by nucleophilic substitution at $\mathrm{C} 1$ of the epoxide ring by one of the terminal nitrogens in hemoglobin. A method for the quantification of the adduct after Edman degradation using GC-MS has already been developed in the middle of the 1990s by Landin et al. who were interested in 2,3-diHOPr-Val as a putative adduct formed by epichlorohydrin (Landin et al. 1996). In different groups of non-smokers, the mean adduct levels were 7.3, 6.8 and $2.1 \mathrm{pmol}$ 2,3-diHOPr-Val/g globin in industrial workers $(n=8)$, clerks of the same German company $(n=3)$ and Swedish control subjects $(n=6)$, respectively. Smokers were found to have distinctly higher mean levels of 21.1, 13.1 and 9.5 pmol 2,3-diHOPr-Val/g globin in industrial workers $(n=7)$, clerks $(n=8)$ and control subjects $(n=4)$, respectively. The authors already speculated-long before the discovery of glycidyl esters in refined oils and fats- that the measurable 2,3-diHOPr-Val levels in the non-smoking population may originate from the intake of dietary glycidol formed by heat treatment of food (Landin et al. 1997). This hypothesis was supported by the observation that 2,3-diHOPr-Val levels were higher in rats fed heated standard chow in comparison to the adduct levels in rats receiving a normal diet (Landin et al. 2000).

Research initiated following the discovery of glycidyl esters in food has already demonstrated the applicability of 2,3-diHOPr-Val as a marker of internal exposure in rodents treated with high doses of orally administered glycidol (Aasa et al. 2017a; Appel et al. 2013; Honda et al. 2014). Surprisingly, the levels of 2,3-diHOPr-Val in blood samples from an observational study with participants exposed to a glycidyl ester-rich vegetable oil did not differ from those not exposed to this oil (Honda et al. 2012). However, the interpretability of the study is limited due to the lack of data on the actual glycidol exposure of the participants.

To prove the applicability of 2,3-diHOPr-Val as a biomarker of internal glycidol exposure in humans, we first developed a novel method for the quantification of 2,3-diHOPr-Val at the $\mathrm{N}$ terminus of hemoglobin. The glycidol adduct was cleaved by fluorescein-5-isothiocyanate (FITC)-mediated Edman degradation (Fig. S1 in the Supplementary Material), enriched by solid-phase extraction and quantified by isotope-dilution ultra-high performance liquid chromatography-mass spectrometry (UHPLC-MS/MS) and multiple reaction monitoring (MRM) (Hielscher et al. 2017). We here report on the results of a controlled exposure in 11 participants eating a glycidyl ester-rich palm fat (containing $8.7 \mathrm{mg}$ ester-bound glycidol per $\mathrm{kg}$ ) over 4 weeks. The levels of 2,3-diHOPr-Val in blood were monitored over this period 
of increased exposure as well as over a "washout" period of 15 weeks thereafter. The aims of this study were firstly to confirm that a high dietary exposure for a limited time is reflected in an increase and a following decrease of the adduct levels (proof of principle), and secondly to estimate the general exposure level from the relation of adduct levels before the intervention ("background") to the adduct levels resulting from the controlled additional exposure using a defined dose.

\section{Materials and methods}

\section{Chemicals}

Glycidol ( $\geq 96 \%$ ), FITC ( $>95 \%$ ), human hemoglobin (lyophilized powder), cyanoacetic acid (99\%) and formic acid ( $\geq 96 \%$ ) were obtained from Sigma-Aldrich (Steinheim, Germany). Potassium hydrogen carbonate, sodium chloride, ammonium hydroxide solution ( $30 \%$ in water), $N, N$-dimethylformamide and acetonitrile (hypergrade) were supplied by Merck (Darmstadt, Germany). Methanol (optigrade) and water (ULC-MS optigrade) were purchased from LGC Promochem (Wesel, Germany). All solvents were of analytical grade. The synthesis of the internal standard 3-(fluorescein5-yl)-1-(2,3-dihydroxypropyl)-5- $d_{7}$-isopropyl-2-thioxo4-imidazolidinone (DHP-Val- $d_{7}-\mathrm{FTH}$ ) has been described previously (Hielscher et al. 2017).

\section{Human exposure study}

The study was conducted at the German Federal Institute for Risk Assessment (BfR) in the Department of Food Safety. Participants were healthy non-smokers of European origin with conventional nutrition habits. The study group consisted of 12 participants (six non-pregnant females and six males). The data of one female participant was excluded from evaluation because she was not able to follow the schedule of blood drawings for the whole study period of 20 weeks. The remaining 11 volunteers had a median age of 30 years (range 23-61), a median height of $1.80 \mathrm{~m}$ (range 1.67-1.90), a median body mass of $72.3 \mathrm{~kg}$ (range $59.6-113.9)$, and a median body mass index of $24.2 \mathrm{~kg} /$ $\mathrm{m}^{2}$ (range 17.6-31.6). Mean body weight was 71.5 and
$78.7 \mathrm{~kg}$ in women and men, respectively. Individual data are provided in Table S1 of the Supplementary Material. The participants consumed a weekly portion of $250 \mathrm{~g}$ palm fat ( $35.7 \mathrm{~g}$ fat daily on average) over 4 weeks. In doing so, they were allowed to replace butter or margarine by the study fat. During the whole study period of 20 weeks (see below), they were otherwise asked to keep their nutritional habits as constant as possible. All participants got a detailed oral consultation about the rationale of the study. They were informed about the carcinogenic potential of glycidol and that the additional exposure to glycidol was well above the mean background exposure, but comparable to the high exposure of formula-fed infants. All participants gave informed consent in writing. The study protocol was approved by the ethics committee of the Charité-Universitätsmedizin Berlin (no. EA4/120/16).

During a study on the contents of bound 2- and 3-monochloropropanediol (2- and 3-MCPD) and glycidol in food items from the German market (BLE 2017), the study fat ("TIP Pflanzenfett", 100\% palm fat according to the label, best by date: 20.11 .2016 ) was identified as the one with the highest level of ester-bound glycidol containing $8.7 \mathrm{mg}$ glycidol $/ \mathrm{kg}$. Food analyses were done by SGS Germany (Hamburg, Germany), using a technique for the indirect determination of ester-bound glycidol in oil matrices. It is based on an alkaline-catalyzed release of glycidol, followed by a transformation to monobromopropanediol according to a modification of the official method Cd 29b-13 ("3-in-1 method") of the American Oil Chemists' Society (AOCS) (Kuhlmann 2016). Consumption of the study fat during the period of 4 weeks led to an additional oral exposure of $8.7 \mathrm{mg}$ bound glycidol (daily amount: $311 \mu \mathrm{g}$ on average). This corresponded to a mean of the individual additional doses of $4.2 \mu \mathrm{g} / \mathrm{kg}$ body weight per day (median 4.3 , range 2.7-5.2), comparable to the current high intake of formulafed infants of $4.9 \mu \mathrm{g} / \mathrm{kg}$ body weight per day (European Food Safety Authority 2016).

\section{Blood samples}

The schedule for blood drawings is depicted in Fig. 1. Two initial samples were taken at an interval of about 1 week. During the exposure period, blood was drawn at the end of
Fig. 1 Study design for the controlled additional exposure with a glycidol ester-rich palm fat and time schedule for the drawings of blood samples

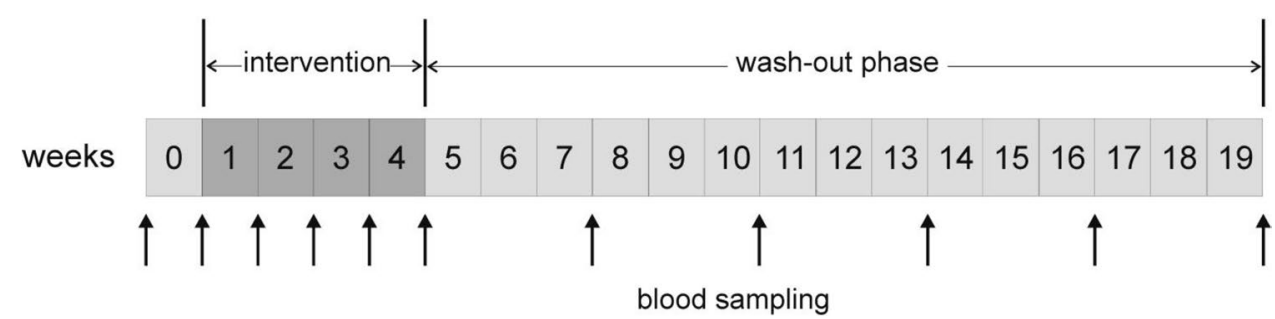


each of the 4 weeks, and every 3 weeks thereafter for additional 15 weeks (total study duration: 20 weeks).

Blood was obtained by puncture of a vein of the antecubital fossa and collected in S-Monovette $4.9 \mathrm{ml} \mathrm{K3E}$ tubes containing ethylenediaminetetraacetic acid (EDTA, $1.6 \mathrm{mg} /$ $\mathrm{ml}$ blood) to prevent coagulation (Sarstedt, Nümbrecht, Germany). The blood was centrifuged at $1500 \times g$ for $12 \mathrm{~min}$ and the plasma was discarded. The erythrocytes were washed three times with $2.5 \mathrm{ml}$ of $0.9 \%$ aqueous sodium chloride and lysed with $2.0 \mathrm{ml}$ water. The hemoglobin concentration was determined with a HemoCue $\mathrm{Hb} 201+$ analyzer (Radiometer, Willich, Germany). The hemoglobin content in the lysed samples varied from 11.8 to $17.5 \mathrm{~g}$ hemoglobin/dl. The samples were stored at $-80{ }^{\circ} \mathrm{C}$ until analysis.

\section{Edman degradation of the $\mathrm{N}$-terminal valine in hemoglobin and solid-phase extraction of DHP-Val-FTH}

The development and validation of the technique for the quantification of 2,3-diHOPr-Val has been published recently (Hielscher et al. 2017). The FITC-mediated Edman degradation for the cleavage of the adduct was conducted as described. Briefly, $250 \mu \mathrm{l}$ aliquots of the reconstituted erythrocytes were mixed with $15 \mu \mathrm{l}$ of $1 \mathrm{M}$ potassium hydrogen carbonate and with $10 \mu \mathrm{l}$ of a $50 \mathrm{nM}$ solution of DHPVal- $d_{7}-$ FTH. The Edman reaction was initiated by addition of $30 \mu \mathrm{l}$ of $428 \mathrm{mM}$ FITC in $N, N$-dimethylformamide. The samples were incubated at $37{ }^{\circ} \mathrm{C}$ and mixed at $800 \mathrm{rpm}$ for $16 \mathrm{~h}$. The analyte was extracted by addition of $1.6 \mathrm{ml}$ acetonitrile. After thorough mixing of the samples, the precipitates of proteins and cell debris were removed by centrifugation $(15,000 \times g, 10 \mathrm{~min})$. The $\mathrm{pH}$ of the supernatants was adjusted by adding $25 \mu \mathrm{l}$ of $1 \mathrm{M}$ aqueous ammonium hydroxide. The analytes were enriched on mixed-mode anion exchange cartridges Oasis MAX $(60 \mathrm{mg}$; Waters, Eschborn, Germany), which were preconditioned with $2 \mathrm{ml}$ of acetonitrile and $2 \mathrm{ml}$ of water. After loading of the samples, the columns were washed with $2 \mathrm{ml}$ each of acetonitrile, water and $0.5 \%$ aqueous cyanoacetic acid. The analytes were eluted with $1.6 \mathrm{ml}$ of $0.25 \%$ cyanoacetic acid dissolved in acetonitrile/water (6:4). The solvents were evaporated under reduced pressure and the samples were reconstituted in $50 \mu \mathrm{l}$ of acetonitrile/water (1:1).

\section{Quantification of DHP-Val-FTH by UHPLC-MS/MS}

The analyses of the Edman degradation samples were conducted using an Acquity UPLC system (Waters) coupled to a QTrap6500 (AB Sciex, Darmstadt, Germany) with an electrospray ionization source operating in the positive ionization mode. Chromatography was performed on a Hypersil GOLD column $(1.9 \mu \mathrm{m}, 2.1 \times 150 \mathrm{~mm}$; Thermo Scientific,
Dreieich, Germany) and the analytes were eluted with a gradient constituted of water (solvent A) and acetonitrile (solvent $\mathrm{B}$ ). Both solvents were acidified with $0.1 \%$ formic acid. The following gradient was applied at a flow rate of $350 \mu \mathrm{l} /$ min: 0-1 min (90\% A), 1-16 min (90-40\% A), 16-17.5 min (0\% A), 17.5-19 min (90\% A). The analyte DHP-Val-FTH and the reference substance DHP-Val- $d_{7}$-FTH were monitored using the MRM mode (Hielscher et al. 2017). Two characteristic fragmentation reactions of the analyte DHPVal-FTH were monitored in parallel to the corresponding transitions of the isotope-labeled standard DHP-Val- $d_{7}-\mathrm{FTH}$. The amount of DHP-Val-FTH was calculated from the peak areas of the signals reflecting the concomitant losses of the isopropyl- and the 2,3-dihydroxyethyl moieties of the analyte $(\mathrm{m} / \mathrm{z}=563 \rightarrow 460)$ and of the deuterated standard $(m / z=570 \rightarrow 460)$. The slightly lower signals caused by elimination of the isopropyl- and the 2,3-dihydroxypropyl groups (DHP-Val-FTH: $m / z=563 \rightarrow 445$, DHP-Val- $d_{7}$-FTH: $m / z=570 \rightarrow 445$ ) were used as qualifier signals. The operating parameters of the mass spectrometer were: curtain gas, 20 psi; collision-activated dissociation (CAD) gas, medium; ion source temperature, $450{ }^{\circ} \mathrm{C}$, ion spray voltage, $5500 \mathrm{~V}$; ion source gas 1, $60 \mathrm{psi}$; ion source gas 2, $50 \mathrm{psi}$. The MRM settings for the monitoring of DHP-Val-FTH and DHP-Val$d_{7}$-FTH are summarized in Table S2 of the Supplementary Material. The data were recorded and analyzed with Analyst 1.6.2 Software (AB Sciex). Peaks were integrated with Multiquant 2.1.1 (AB Sciex).

\section{Calculation of 2,3-diHOPr-Val levels in hemoglobin samples}

The levels of 2,3-diHOPr-Val (A) determined in the blood samples of study participants were calculated as follows:

$A=\frac{\frac{P_{\text {anal }}}{P_{\mathrm{IS}}} \times \text { amount }_{\mathrm{IS}}(\mathrm{pmol})}{\text { amount }_{\mathrm{Hb}}(\mathrm{g})} \times \mathrm{cf}$,

with the peak areas of the quantifier signals of the analyte DHP-Val-FTH $\left(P_{\text {anal }}, m / z=563 \rightarrow 460\right)$ and of the internal standard DHP-Val- $d_{7}$-FTH $\left(P_{\mathrm{IS}}, m / z=570 \rightarrow 460\right)$, with the quantities of the internal standard (amount ${ }_{\mathrm{IS}}$ ) and of hemoglobin $\left(\mathrm{amount}_{\mathrm{Hb}}\right.$ ) used for the Edman degradation, and a conversion factor (cf) reflecting the yield of the Edman degradation. This was determined using the synthetic peptide $N$-(2,3-dihydroxypropyl)-Val-Leu-anilide (DHP-ValLeu-An). The synthesis of the dipeptide DHP-Val-Leu-An is described in the Supplementary Material. The Edman degradation samples were prepared from $250 \mu$ aliquots of human erythrocytes. They were spiked with seven different amounts of the dipeptide standard DHP-Val-Leu-An between 100 and 10,000 fmol and a constant amount of $500 \mathrm{fmol}$ 
DHP-Val- $d_{7}$-FTH in each sample. After the cleavage reaction, the quotient of the peak areas of the quantifier signals of DHP-Val-FTH and DHP-Val- $d_{7}$-FTH were correlated with the amount of DHP-Val-Leu-An in the samples. The yield of the modified Edman degradation (78.1\%) was calculated from the slope of the data after fitting by linear regression (Fig. S2 of the Supplementary Material).

\section{Statistical analysis and modeling}

For the modeling of the time course of 2,3-diHOPr-Val concentration in hemoglobin, it was assumed that the amount of hemoglobin available for adduct formation is always much higher than the amount of glycidol present. Hence, 2,3-diHOPr-Val formation is proportional to the amount of glycidol uptake. However, a part of the adducts is permanently removed due to the limited lifetime of erythrocytes, and in case of constant exposure over time, steady-state conditions are reached after one lifetime. Equations describing the adduct levels resulting from these assumptions have been already published earlier (Fennell et al. 1992). We amended them by a term considering the adduct level representing the background exposure (Fig. S3 of the Supplementary Material) and assumed that this level is constant during the study period.

The 2,3-diHOPr-Val concentration was fitted to the measured values using a population approach (mixed model) where all the parameters, namely the background adduct level bg, the adduct lifetime $\tau$, and the proportionality constant $k$ [increase of adduct level per dose on a body weight basis, corresponding to the so-called hemoglobin binding index (Neumann 1984)], were considered to be random factors. Several models were fitted with these parameters as either fixed or random; finally, the best fitting model based on the Akaike information criterion (AIC) was chosen. Fitting separate models for each individual and using the resulting parameter distributions will give similar results, but the approach chosen allows estimation of parameter distributions in the population from an integrated inspection of the data set.

One of the possible uses of the model is the estimation of long-term glycidol exposure, assumed to be solely due to a person's diet, based on 2,3-diHOPr-Val measurements. Using the steady-state model equation $H=D \cdot \tau \cdot k / 2$ for the hemoglobin adduct level $H$ in the steady-state condition depending on the dose $D$, the inverse model equation:

$D=\frac{2}{\tau \cdot k} \cdot H=a \cdot H$

can be derived.

Based on the inverse model, a Monte Carlo simulation was applied to obtain a confidence interval for the exposure estimate. For modeling, the R statistics language (R Core
Team 2018) was used with the 'nlme' (nonlinear mixed effects) package (Pinheiro et al. 2018). The R code which was used for the computations is provided in the Supplementary Material.

\section{Results}

\section{Quantification of 2,3-diHOPr-Val in human erythrocytes}

The 2,3-diHOPr-Val levels in blood samples from 11 individuals were determined after FITC-mediated Edman degradation by UHPLC-MS/MS MRM. The exemplary chromatograms of a DHP-Val-FTH analysis from one of the participants show the increasing 2,3-diHOPr-Val levels after weeks 1 and 4 in comparison to the background level before the start of the intervention period (Fig. 2). The presence of two peaks was hypothesized to originate from the formation of two diastereomers of 2,3-diHOPr-Val by nucleophilic attack of the nitrogen of the $\mathrm{N}$-terminal valines in hemoglobin at the $\mathrm{C} 1$ in the epoxide ring of glycidol (Hielscher

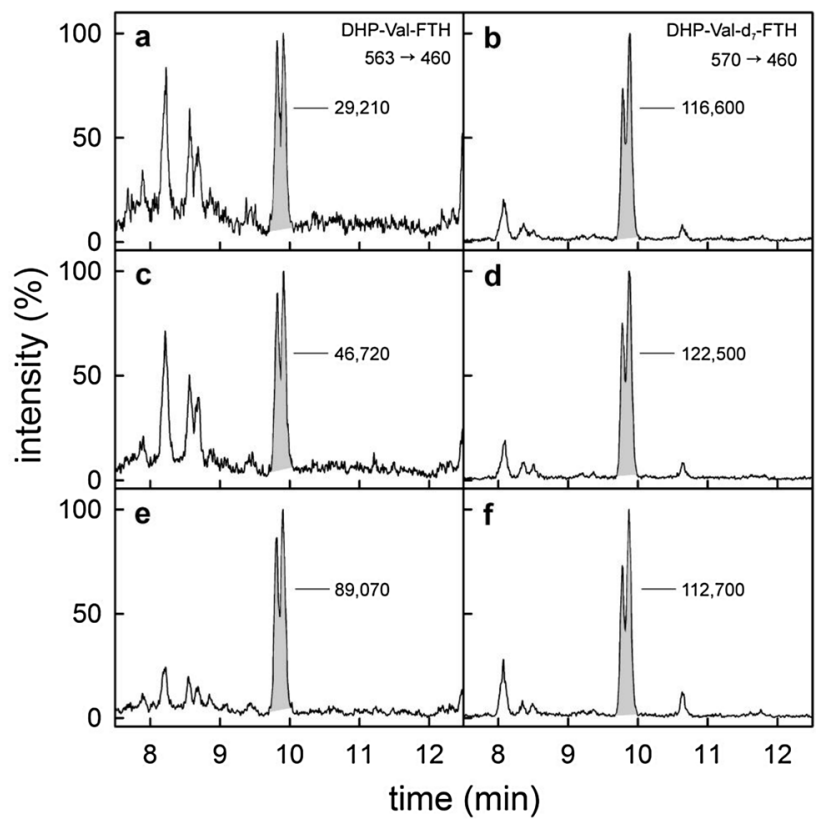

Fig. 2 UHPLC-MS/MS chromatograms of FITC-mediated Edman degradation samples of one study participant before the intervention period $(\mathbf{a}, \mathbf{b})$, after week $1(\mathbf{c}, \mathbf{d})$ and after week 4 of the intervention period $(\mathbf{e}, \mathbf{f})$. The left panels depict the chromatograms of the quantifier trace of DHP-Val-FTH with the fragmentation $\mathrm{m} / z=563 \rightarrow 460$. The chromatograms on the right hand side were from the transitions $m / z=570 \rightarrow 460$ of the internal isotope-labeled standard DHP-Val$d_{7}$-FTH used for the quantification of DHP-Val-FTH. The mass spectrometric signal intensity was normalized to $100 \%$. The peaks areas (au) of DHP-Val-FTH and of DHP-Val- $d_{7}$-FTH denoted in the chromatograms reflect the relative signal intensities 
et al. 2017). In this former investigation, the yield of the Edman degradation was not considered. Thus, the background levels in the current work are slightly higher compared to those reported before.

\section{2,3-DiHOPr-Val in blood samples in the course of the controlled exposure to palm fat}

During the study period of 20 weeks, 11 blood samples were drawn from each of the participants (Fig. 1). The results of individual 2,3-diHOPr-Val analyses are summarized in Table S3 of the Supplementary Material. The means of duplicates are displayed in Fig. 3. Two background adduct levels determined about 1 week apart before the start of the intervention period were in the range of 2.9-5.5 pmol 2,3-diHOPr-Val/g hemoglobin, with only little differences for the results of individuals (mean \pm SD $4.1 \pm 0.8$ and $4.0 \pm 0.9 \mathrm{pmol} 2,3-\mathrm{diHOPr}-\mathrm{Val} / \mathrm{g}$ hemoglobin, respectively). During the daily consumption of palm fat, mean adduct levels $( \pm \mathrm{SD})$ continuously increased to $6.6 \pm 1.1 \mathrm{pmol} 2,3-\mathrm{diHOPr}-\mathrm{Val} / \mathrm{g}$ hemoglobin after 1 week, to $8.8 \pm 1.2 \mathrm{pmol} 2,3-\mathrm{diHOPr}-\mathrm{Val} / \mathrm{g}$ hemoglobin after 2 weeks, to $10.9 \pm 1.7 \mathrm{pmol} 2,3$-diHOPr-Val/g hemoglobin after 3 weeks, and to $12.2 \pm 1.7 \mathrm{pmol} 2,3-\mathrm{diHOPr}-\mathrm{Val} / \mathrm{g}$ hemoglobin after 4 weeks. Each weekly increase was highly significant $(p<0.001$, pairwise Student's $t$ test), but the weekly mean increments decreased continuously from $2.6 \mathrm{pmol}$ 2,3-diHOPr-Val/g hemoglobin in the first week to $1.3 \mathrm{pmol} 2,3-\mathrm{diHOPr}-\mathrm{Val} / \mathrm{g}$ hemoglobin in the fourth week. Individual 4-week increments were between 5.4 and

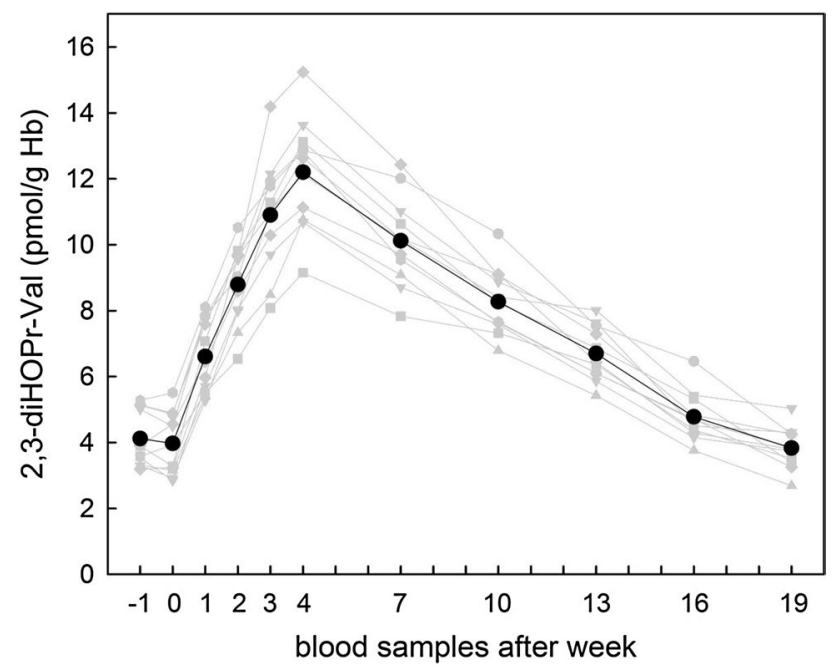

Fig. 3 Levels of 2,3-diHOPr-Val in hemoglobin samples from the 11 participants before, during and after the intervention period with consumption of about $36 \mathrm{~g}$ palm fat per day containing $8.7 \mathrm{mg}$ glycidol/ $\mathrm{kg}$ over 4 weeks (grey symbols, with respective means for each point of time as black dots)
$10.2 \mathrm{pmol}$ 2,3-diHOPr-Val/g hemoglobin, and correlated negatively with body weights (Pearson, $r^{2}=0.28$ ).

During the following washout period of 15 weeks, the adduct levels continuously declined (Fig. 3), reaching a mean level of $3.8 \pm 0.6 \mathrm{pmol} 2,3$-diHOPr-Val/g hemoglobin, not significantly different from the start level of $4.0 \pm 0.9 \mathrm{pmol}$ 2,3-diHOPr-Val/g hemoglobin. The decline was more or less linear with an average daily rate of $-0.080 \mathrm{pmol}$ 2,3-diHOPr-Val/g hemoglobin ( $0.95 \%$ of the mean increment of $8.2 \mathrm{pmol} 2,3$-diHOPr-Val/g hemoglobin).

\section{Simulation of the time courses of 2,3-diHOPr-Val}

To determine the distribution of $k$ and $\tau$ in the population, the data of all study participants were analyzed using a nonlinear mixed model. The model which fitted the data best was the one having all three parameters $(k, \tau, \mathrm{bg})$ as random parameters. Detailed results of the statistical evaluation are, together with the $\mathrm{R}$ code, provided as supplemental information. The parameter estimates $( \pm$ SE) were $k=0.082 \pm 0.004 \mathrm{pmol} 2,3-\mathrm{diHOPr}-\mathrm{Val} / \mathrm{g}$ hemoglobin per $\mu \mathrm{g}$ glycidol $/ \mathrm{kg}$ body weight and $\tau=104 \pm 5$ days. The average background level of the adducts was $4.0 \pm 0.2 \mathrm{pmol}$ 2,3-diHOPr-Val/g hemoglobin. Individual realizations of the parameters for each participant were also computed (Table 1). The values of $k$ were in a range between 0.061 and $0.099 \mathrm{pmol} 2,3$-diHOPr-Val/g hemoglobin per $\mu \mathrm{g}$ glycidol/kg body weight. There was no significant difference between the adduct level/dose ratio $(k)$ of males and females. The lifetime of the adducts $(\tau)$ was between 76 and 133 days. The variation of the individual curves (solid line) around the mean (dashed line) is visualized in Fig. 4.

The estimate for the parameter in the inverse model was $a=0.235 \mu \mathrm{g}$ glycidol $/ \mathrm{kg}$ body weight $/$ day per $\mathrm{pmol}$ 2,3-diHOPr-Val/g hemoglobin (95\% confidence interval $0.164,0.364)$. For the mean background level of $\mathrm{H}=4.0 \mathrm{pmol} 2,3-\mathrm{diHOPr}-\mathrm{Val} / \mathrm{g}$ hemoglobin, a daily glycidol dose of $D=a \cdot H=0.94 \mu \mathrm{g} / \mathrm{kg}$ body weight $(95 \%$ confidence interval: $0.66,1.45 \mu \mathrm{g} / \mathrm{kg}$ body weight) was estimated (individual values ranging from 0.63 to $1.52 \mu \mathrm{g} /$ $\mathrm{kg}$ body weight, Table 1 ). The mean additional study dose of glycidol of $4.2 \mu \mathrm{g} / \mathrm{kg}$ body weight corresponded to a mean increase of the adduct level under steady-state conditions of $H=D / a=18.0$ pmol 2,3-diHOPr-Val/g hemoglobin.

\section{Discussion}

In this exposure study with 11 participants, we were able to confirm that an additional dietary exposure to fatty acid esters of glycidol for 4 weeks is indeed reflected in a corresponding increase of the levels of the hemoglobin adduct 2,3-diHOPr-Val during this time, followed by a 
Table 1 Results of fitting analyses of individual data sets with the nonlinear mixed model describing the time courses of the adduct levels over the intervention period of 4 weeks and the following washout period of 15 weeks: adduct increment factors $(k)$, adduct lifetimes $(\tau)$ and individual background levels of 2,3-diHOPr-Val (bg)

\begin{tabular}{llcll}
\hline Participant & $\begin{array}{l}k \text { (pmol 2,3-diHOPr-Val/g Hb per } \\
\mu \text { g glycidol/kg body weight) }\end{array}$ & $\begin{array}{l}\tau \text { (days) } \\
\text { A }\end{array}$ & $\begin{array}{l}\text { bg (pmol } \\
2,3-\text { diHOPr-Val/g } \\
\text { Hb) }\end{array}$ & $\begin{array}{l}D \text { ( } \mu \text { glycidol/ } \\
\text { kg body weight) }\end{array}$ \\
\hline A & 0.071 & 105 & 3.54 & 0.95 \\
B & 0.082 & 113 & 3.77 & 0.81 \\
C & 0.078 & 133 & 3.24 & 0.63 \\
D & 0.097 & 105 & 3.66 & 0.72 \\
E & 0.096 & 105 & 3.96 & 0.79 \\
F & 0.099 & 93 & 4.67 & 1.01 \\
G & 0.074 & 98 & 4.58 & 1.26 \\
H & 0.061 & 101 & 4.03 & 1.31 \\
I & 0.079 & 112 & 4.74 & 1.07 \\
J & 0.080 & 106 & 3.21 & 0.76 \\
K & 0.088 & 76 & 5.09 & 1.52 \\
\hline
\end{tabular}

From these data, the individual background doses $D$ were calculated using the steady-state model equation $H=b g=D \cdot \tau \cdot k / 2$

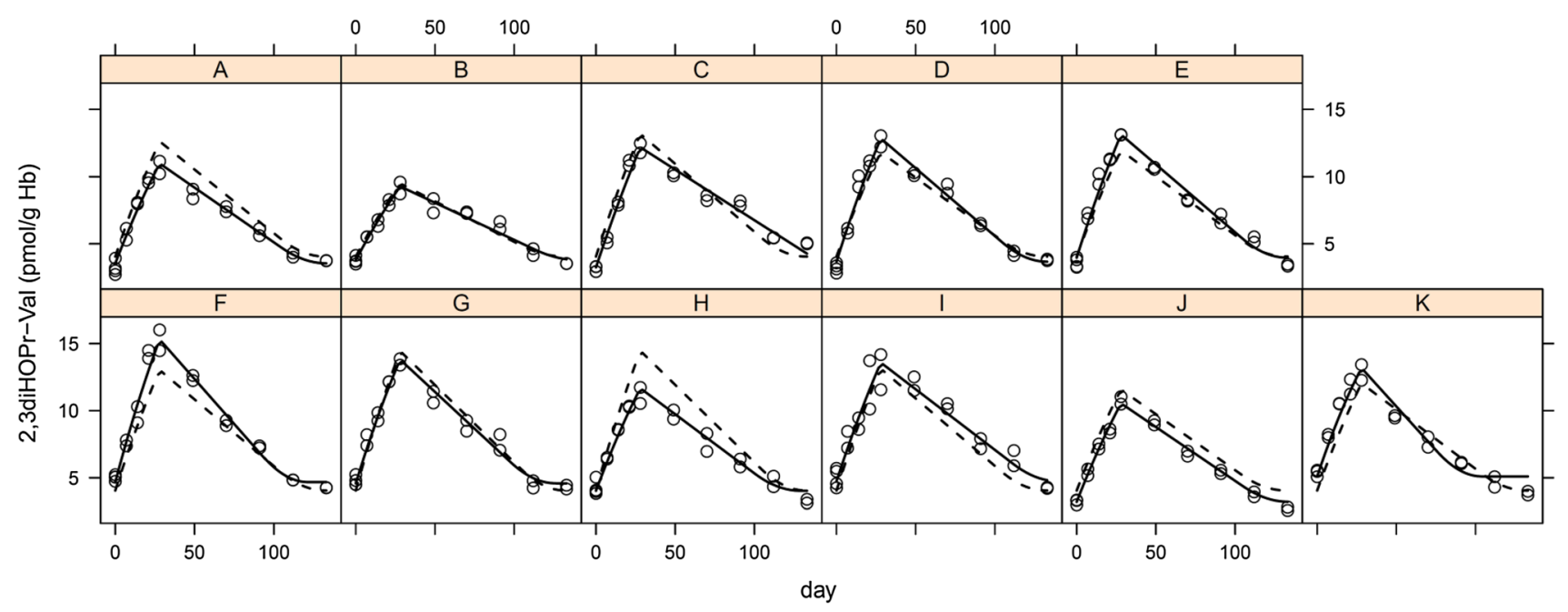

Fig. 4 Separate kinetics of 2,3-diHOPr-Val levels in blood samples of the 11 participants. The time course of adduct levels in each of the participants was fitted using the model described in the method section (black lines). The fit of the cumulative data from all participants

decrease-according to the lifetime of erythrocytes-back to the levels before the intervention period (first aim of the study: proof of principle). The unambiguous result (Figs. 3, 4) was achieved by the daily consumption of about $36 \mathrm{~g}$ of a glycidol ester-rich palm fat, resulting in an additional mean glycidol exposure of $4.2 \mu \mathrm{g} / \mathrm{kg}$ body weight per day, well above the mean background exposure across surveys and age groups estimated to be between 0.1 and $0.9 \mu \mathrm{g} / \mathrm{kg}$ body weight per day in Europe, but not much below the current high intake of formula-fed infants of $4.9 \mu \mathrm{g} / \mathrm{kg}$ body weight per day (European Food Safety Authority 2016). The duration of the controlled exposure was chosen to be 4 weeks to significantly increase the expected adduct level on the one hand, but not to unnecessarily prolong the additional is added for the sake of comparison (dashed line). Please note that the latter lines vary in height depending on the respective individual body weight

glycidol exposure on the other hand: after 4 weeks of continuous exposure, adduct level are expected to reach $40-50 \%$ of the steady-state levels, depending on the lifetime of erythrocytes [(Fennell et al. 1992), and Fig. S4 of the Supplementary Material].

The oral administration of glycidol or fatty acid esters of glycidol has already been shown to proportionally increase the levels of 2,3-diHOPr-Val in rats (Appel et al. 2013; Honda et al. 2014) and mice (Aasa et al. 2017a), and therefore demonstrates its suitability as biomarker of exposure in rodents. Surprisingly, the only study in humans failed to detect higher levels of 2,3-diHOPr-Val in blood samples from subjects exposed to a diacylglycerol oil with a relatively high content of glycidol fatty acid esters in a cafeteria 
and due to the use for cooking at home, compared to subjects not exposed to this oil (Honda et al. 2012). However, the interpretability of the study is limited due to the missing control of the exposure to fatty acid esters of glycidol in both groups.

\section{The kinetics of formation and removal of adducts}

With the controlled exposure using a glycidol ester-rich palm fat, we observed a distinct increase of the levels of 2,3-diHOPr-Val in the blood samples of the participants. Modeling of the data allowed deriving individual values for the increase of adduct levels per dose on a body weight basis $(k)$. The increase of the mean adduct levels was most pronounced in the first week and became continuously smaller from week 2 to week 4 , although the daily glycidol dose was constant. The slightly hyperbolical increase of adduct levels indicated the expected growing influence of adduct removal due to the limited lifetime of erythrocytes (see also Fig. S4 of the Supplementary Material). This became more pronounced after the intervention period, leading to a continuous decrease of the 2,3-diHOPr-Val levels with an apparent zero-order kinetics (Fennell et al. 1992). The mean lifetime of the adducts ( $\tau=104$ days) determined by the fitting of all data sets is within the range observed for the lifetime of human erythrocytes, e.g., $110 \pm 21$ days (Bentley et al. 1974) or $116 \pm 15$ days (Mock et al. 1999), indicating that 2,3-diHOPr-Val is a stable adduct cleared together with the erythrocyte at the end of its lifetime.

\section{Estimate of the glycidol exposure from the adduct level}

The second aim of our study was to estimate the general exposure level from the relation of adduct levels before the intervention period ("background", bg) to the adduct levels, resulting from the controlled additional exposure using a defined daily dose. The calculated mean daily glycidol exposure determined from the adduct levels of the participants before the onset of the intervention period (mean $4.0 \mathrm{pmol}$ 2,3-diHOPr-Val/g hemoglobin) was $0.94 \mu \mathrm{g}$ glycidol $/ \mathrm{kg}$ body weight (95\% confidence interval: $0.66,1.45)$. This is considerably higher than the median value of the mean chronic exposure to glycidol from esters across dietary surveys for adults of $0.2 \mu \mathrm{g}$ glycidol $/ \mathrm{kg}$ body weight, estimated for European countries by the EFSA (European Food Safety Authority 2016). Preliminary results from a more recent exposure estimate for adults in Germany suggest that the mean dietary exposure is at least fourfold below the biomarker-based confidence interval of the exposure estimate (BfR, unpublished data). The deviation between the dose calculated from our study data and the estimated external exposure via food may have different reasons. The latter may underestimate the intake, as possibly not all foods with relevant levels of glycidyl esters are currently identified and analyzed to the necessary extent. In addition, formation of glycidol during domestic food preparation may play a role, as well as inhalational exposure, e.g., from passive smoking. Theoretically, an endogenous formation may also contribute to the internal exposure (Honda et al. 2012, 2014).

\section{Specificity of the adduct 2,3-diHOPr-Val for glycidol}

Furthermore, 2,3-diHOPr-Val may not be specific for the internal exposure to glycidol. Several other substances, i.e., 3-MCPD and epichlorohydrin (Landin et al. 1997) as well as allyl alcohol (Honda et al. 2012), were discussed as possible precursors of 2,3-diHOPr-Val. However, experimental data indicated that these compounds may not contribute significantly to the formation of 2,3-diHOPr-Val. The adduct levels in blood samples of industry workers exposed to epichlorohydrin were not higher compared to those of non-exposed clerks (Landin et al. 1997). Also, 2,3-diHOPr-Val was not detected (limit of detection $=10 \mathrm{pmol} / \mathrm{g}$ globin) in human blood samples of emergency responders and of residents living close to the accidental site of a train accident in $\mathrm{Bad}$ Münder (Germany) in 2002, in which > 30 tons of epichlorohydrin was spilt (Wollin et al. 2014). In rats, the 2,3-diHOPr$\mathrm{Val} /$ dose ratio determined after intraperitoneal administration of epichlorohydrin was about $1.1 \times 10^{-3} \mathrm{pmol} / \mathrm{g}$ globin per $\mu$ g glycidol $/ \mathrm{kg}$ body weight, which is about 230 times (Appel et al. 2013) and about 50 times (Honda et al. 2014) lower than the adduct level/dose ratios observed after treatment of rats with glycidol. A recent study in female BalbC mice showed that 2,3-diHOPr-Val was indeed formed following administration of 3-MPCD, albeit with a mere increment of $0.035 \times 10^{-3} \mathrm{pmol} / \mathrm{g}$ hemoglobin per $\mu \mathrm{g} 3$-MCPD/ $\mathrm{kg}$ body weight (Aasa et al. 2017b). This is about 550fold lower than the adduct level/dose ratio observed after treatment of female BalbC mice with glycidol (Aasa et al. 2017a). In summary, there are no indications so far of reactive substances which form the adduct 2,3-diHOPr-Val in a relevant amount.

\section{Summary and conclusions}

2,3-diHOPr-Val is a hemoglobin adduct of glycidol in humans and animals. Using a modified Edman degradation and UHPLC-MS/MS, the adduct can reliably be quantified even in the low pmol per $\mathrm{g}$ hemoglobin range resulting from the background exposure of glycidol (Hielscher et al. 2017). Interpretation of such levels as biomarker of internal exposure regarding the corresponding level of external exposure requires information about the ratio of adduct level to external dose and the actual lifetime of the adduct. 
Therefore, we performed a controlled study with 11 participants who consumed a relatively high daily amount of a glycidol ester-rich palm fat during a 4-week intervention period. Frequent blood sampling was performed to monitor the 2,3-diHOPr-Val adduct levels during formation and following removal, allowing to finally study the kinetics using a nonlinear mixed model.

In our study, we were able to demonstrate for the first time that the relatively high oral exposure during the intervention period was reflected in corresponding distinct increases of 2,3-diHOPr-Val levels in all 11 participants, following the slope expected over time. Modeling allowed determining values for the adduct level/dose ratio $(k)$ and the adduct lifetime $(\tau)$. The adduct was shown to be stable with a mean lifetime of 104 days, very likely corresponding to the mean lifetime of the erythrocytes in our study. Therefore, 2,3-diHOPr-Val was proven to be a biomarker of the external dietary exposure to fatty acid esters of glycidol. Interindividual variability was generally low (SD/mean 14 and $13 \%$ for $k$ and $\tau$, respectively), most likely due to passive mechanisms and missing relevance of metabolism apart from the gastrointestinal cleavage of the esters during digestion, assumed to be nearly $100 \%$ in humans.

Assuming steady-state conditions, the low interindividual variability, in general, allows to estimate a value for a corresponding external exposure level from a single blood sample for the period of about 3 months before the sampling, simply via backcalculation of the 2,3-diHOPr-Val level using the mean value of $a=0.235 \mu \mathrm{g}$ glycidol $/ \mathrm{kg}$ body weight/day per pmol 2,3-diHOPr-Val/g hemoglobin (variability SD/mean in our study 19\%). This approach can be used for groups of non-smokers (e.g., to monitor the time trend) or for nonsmoking individuals, even if the individual values of $k$ and $\tau$ are not known.

In doing so for the background adduct levels of the participants of our study, the estimate for the mean external glycidol exposure was $0.94 \mu \mathrm{g} / \mathrm{kg}$ body weight. This value is considerably higher than the current estimates for adults using occurrence and consumption data of food. The possible reasons for this discrepancy are discussed above (other oral or inhalational glycidol sources, endogenous formation, exposure to other chemicals also forming the adduct 2,3-diHOPr-Val). However, these possibilities are currently not convincing to plausibly explain such a big discrepancy. Therefore, further research is necessary to confirm our results and to clarify the issue (e.g., by investigating the internal exposure of subjects avoiding any consumption of heated food). Nevertheless, the possibility of an internal exposure level of glycidol much higher than expected from conventional estimation of external exposure via food should be taken seriously in risk assessment, as finally the internal dose-independently of its source—triggers possible effects.
Acknowledgements The authors thank Dr. Jan Kuhlmann (SGS Germany GmbH, Hamburg, Germany) for the identification of the palm fat with a high content of glycidyl esters and for the subsequent purchase of a quantity of the same batch adequate to perform the study. Furthermore, we thank Corinna Genrich and Christel Rozycki for their excellent technical assistance.

\section{Compliance with ethical standards}

Conflict of interest The authors declare that they have no conflicts of interest.

Open Access This article is distributed under the terms of the Creative Commons Attribution 4.0 International License (http://creativeco mmons.org/licenses/by/4.0/), which permits unrestricted use, distribution, and reproduction in any medium, provided you give appropriate credit to the original author(s) and the source, provide a link to the Creative Commons license, and indicate if changes were made.

\section{References}

Aasa J, Abramsson-Zetterberg L, Carlsson H, Törnqvist M (2017a) The genotoxic potency of glycidol established from micronucleus frequency and hemoglobin adduct levels in mice. Food Chem Toxicol 100:168-174

Aasa J, Törnqvist M, Abramsson-Zetterberg L (2017b) Measurement of micronuclei and internal dose in mice demonstrates that 3-monochloropropane-1,2-diol (3-MCPD) has no genotoxic potency in vivo. Food Chem Toxicol 109:414-420

Appel KE, Abraham K, Berger-Preiss E et al (2013) Relative oral bioavailability of glycidol from glycidyl fatty acid esters in rats. Arch Toxicol 87:1649-1659

Bentley SA, Lewis SM, White JM (1974) Red cell survival studies in patients with unstable haemoglobin disorders. Br J Haematol 26:85-92

European Food Safety Authority (2016) Risks for human health related to the presence of 3- and 2-monochloropropanediol (MCPD), and their fatty acid esters, and glycidyl fatty acid esters in food. EFSA J 14:4426-4584

Federal Institute of Risk Assessment (BfR) (2009) Initial evaluation of the assessment of levels of glycidol fatty acid esters detected in refined vegetable fats, BfR Opinion No. 007/2009. Federal Institute of Risk Assessment (BfR), Berlin

Fennell TR, Sumner SC, Walker VE (1992) A model for the formation and removal of hemoglobin adducts. Cancer Epidemiol Biomark Prev 1:213-219

Hielscher J, Monien BH, Abraham K, Jessel S, Seidel A, Lampen A (2017) An isotope-dilution UPLC-MS/MS technique for the human biomonitoring of the internal exposure to glycidol via a valine adduct at the $\mathrm{N}$-terminus of hemoglobin. J Chromatogr B 1059:7-13

Honda H, Fujii K, Yamaguchi T, Ikeda N, Nishiyama N, Kasamatsu T (2012) Glycidol exposure evaluation of humans who have ingested diacylglycerol oil containing glycidol fatty acid esters using hemoglobin adducts. Food Chem Toxicol 50:4163-4168

Honda H, Törnqvist M, Nishiyama N, Kasamatsu T (2014) Characterization of glycidol-hemoglobin adducts as biomarkers of exposure and in vivo dose. Toxicol Appl Pharmacol 275:213-220

Inagaki R, Hirai C, Shimamura Y, Masuda S (2016) Formation of glycidol fatty acid esters in meat samples cooked by various methods. J Food Process Technol 7:557-562 
International Agency for Research on Cancer (2000) Glycidol in some industrial chemicals, vol 77. International Agency for Research on Cancer, Lyon

Kuhlmann J (2016) Analysis and occurrence of dichloropropanol fatty acid esters and related process-induced contaminants in edible oils and fats. Eur J Lipid Sci Technol 118:382-395

Landin HH, Osterman-Golkar S, Zorcec V, Törnqvist M (1996) Biomonitoring of epichlorohydrin by hemoglobin adducts. Anal Biochem 240:1-6

Landin HH, Grummt T, Laurent C, Tates A (1997) Monitoring of occupational exposure to epichlorohydrin by genetic effects and hemoglobin adducts. Mutat Res 381:217-226

Landin HH, Tareke E, Rydberg P, Olsson U, Törnqvist M (2000) Heating of food and haemoglobin adducts from carcinogens: possible precursor role of glycidol. Food Chem Toxicol 38:963-969

Mock DM, Lankford GL, Widness JA, Burmeister LF, Kahn D, Strauss RG (1999) Measurement of red cell survival using biotin-labeled red cells: validation against $51 \mathrm{Cr}$-labeled red cells. Transfusion (Paris) 39:156-162

National Toxicology Program (1990) Toxicology and carcinogenesis studies of glycidol (CAS No. 556-52-5) in F344/N rats and B6C3F1 mice (Gavage Studies). Natl Toxicol Program Tech Rep Ser 374:1-229

\section{Affiliations}

\section{Klaus Abraham ${ }^{1} \cdot$ Jan Hielscher ${ }^{1} \cdot$ Tobias Kaufholz $^{2} \cdot$ Hans Mielke $^{2} \cdot$ Alfonso Lampen $^{1} \cdot$ Bernhard Monien $^{1}$}

1 Department of Food Safety, German Federal Institute for Risk Assessment (BfR), Max-Dohrn-Str. 8-10, 10589 Berlin, Germany
Neumann HG (1984) Analysis of hemoglobin as a dose monitor for alkylating and arylating agents. Arch Toxicol 56:1-6

Pinheiro J, Bates D, DebRoy S, Sarkar D, Team RC (2018) nlme: linear and nonlinear mixed effects models. R package version 3.1-131.1

R Core Team (2018) R: a language and environment for statistical computing. R Foundation for Statistical Computing, Vienna

Slott VL, Hales BF (1985) Teratogenicity and embryolethality of acrolein and structurally related compounds in rats. Teratology 32:65-72

Svejkovska B, Novotny O, Divinova V, Reblova Z, Dolezal M, Velisek J (2004) Esters of 3-chloropropane-1,2-diol in foodstuffs. Czech J Food Sci 22:190-196

Wakabayashi K, Kurata Y, Harada T, Tamaki Y, Nishiyama N, Kasamatsu T (2012) Species differences in toxicokinetic parameters of glycidol after a single dose of glycidol or glycidol linoleate in rats and monkeys. J Toxicol Sci 37:691-698

Wollin KM, Bader M, Müller M, Lilienblum W, Csicsaky M (2014) Assessment of long-term health risks after accidental exposure using haemoglobin adducts of epichlorohydrin. Toxicol Lett 231:378-386
2 Department of Exposure, German Federal Institute for Risk Assessment (BfR), Max-Dohrn-Str. 8-10, 10589 Berlin, Germany 